\title{
Identification of Diabetic Retinopathy Genes through a Genome-Wide Association Study among Mexican-Americans from Starr County, Texas
}

\author{
Yi-Ping Fu, ${ }^{1}$ D. Michael Hallman, ${ }^{1}$ Victor H. Gonzalez, ${ }^{2}$ Barbara E. K. Klein, ${ }^{3}$ \\ Ronald Klein, ${ }^{3}$ M. Geoffrey Hayes, ${ }^{4}$ Nancy J. Cox, ${ }^{5}$ Graeme I. Bell, ${ }^{5}$ and Craig L. Hanis ${ }^{1}$ \\ ${ }^{1}$ Human Genetics Center, School of Public Health, The University of Texas Health Science Center at Houston, \\ P.O. Box 20186, Houston, TX 77225, USA \\ ${ }^{2}$ Valley Retina Institute, McAllen, TX 78503, USA \\ ${ }^{3}$ Department of Ophthalmology and Visual Sciences, University of Wisconsin School of Medicine and Public Health, \\ Madison, WI 53705, USA \\ ${ }^{4}$ Division of Endocrinology, Feinberg School of Medicine, Northwestern University, Chicago, IL 60611, USA \\ ${ }^{5}$ Department of Human Genetics, The University of Chicago, Chicago, IL 60637, USA
}

Correspondence should be addressed to Craig L. Hanis, craig.l.hanis@uth.tmc.edu

Received 18 December 2009; Revised 22 May 2010; Accepted 14 July 2010

Academic Editor: Kavita Hegde

Copyright (C) 2010 Yi-Ping Fu et al. This is an open access article distributed under the Creative Commons Attribution License, which permits unrestricted use, distribution, and reproduction in any medium, provided the original work is properly cited.

\begin{abstract}
To identify genetic loci for severe diabetic retinopathy, 286 Mexican-Americans with type 2 diabetes from Starr County, Texas, completed physical examinations including fundus photography for diabetic retinopathy grading. Individuals with moderate-tosevere non-proliferative and proliferative diabetic retinopathy were defined as cases. Direct genotyping was performed using the Affymetrix GeneChip Human Mapping $100 \mathrm{~K}$ Set, and SNPs passing quality control criteria were used to impute markers available in HapMap Phase III Mexican population (MXL) in Los Angeles, California. Two directly genotyped markers were associated with severe diabetic retinopathy at a $P$-value less than .0001 : SNP rs2300782 $\left(P=6.04 \times 10^{-5}\right)$ mapped to an intron region of CAMK4 (calcium/calmodulin-dependent protein kinase IV) on chromosome 5, and SNP rs10519765 $\left(P=6.21 \times 10^{-5}\right)$ on chromosomal $15 \mathrm{q} 13$ in the FMN1 (formin 1) gene. Using well-imputed markers based on the HapMap III Mexican population, we identified an additional 32 SNPs located in 11 chromosomal regions with nominal association with severe diabetic retinopathy at $P$-value less than .0001. None of these markers were located in traditional candidate genes for diabetic retinopathy or diabetes itself. However, these signals implicate genes involved in inflammation, oxidative stress and cell adhesion for the development and progression of diabetic retinopathy.
\end{abstract}

\section{Introduction}

Diabetic retinopathy is a common microvascular complication of diabetes and remains one of the leading causes of blindness throughout the world [1]. It is estimated that 4.1 million Americans have diabetic retinopathy, which causes 12,000 to 24,000 new cases of blindness every year [2]. National Health Interview Survey and US census data lead to projections that the number of Americans 40 years or older having diabetic retinopathy will triple from 5.5 millions in 2005 to 16 millions in 2050 [3]. Although the underlying mechanisms leading to diabetic retinopathy have not been clarified, many risk factors have been reported, including poor glycemic control, longer diabetes duration, hypertension, hyperlipidemia, and albuminuria [4-7]. Evidence from ethnic and family studies has implicated genetic susceptibility for diabetic retinopathy. Mexican-Americans and African-Americans have been reported to have higher prevalence and worse severity of diabetic retinopathy in the US population when compared to non-Hispanic Whites [8, 9]. The Diabetes Control and Complications Trial (DCCT) showed a 3.1 times increased risk of severe retinopathy 
for individuals with retinopathy-positive relatives, and a correlation of retinopathy severity of 0.187 for all family members [10]. Similar familial clustering of diabetic retinopathy was also found among South Indians [11] and Mexican-Americans [12] with type 2 diabetes. The FINDEye study recently reported the broad sense heritability for diabetic retinopathy as $27 \%$ overall and $24 \%$ in MexicanAmerican families [13].

The suggestive genetic contribution to diabetic retinopathy has lead to the search for candidate genes and for genome-wide linkage between genetic markers and this complex disease, but no conclusive loci have been identified or replicated [14, 15]. Affected sibpair analysis in Pima Indians showed some evidence for linkage to diabetic retinopathy on chromosome 3 and 9 [16], and another signal on chromosome 1 was found in a subsequent study of the same population [17]. Our group also performed a linkage scan in Mexican-Americans from Starr County, Texas, and proposed 25 potential candidate genes for diabetic retinopathy under the linkage peaks on chromosome 3, 6, 12, 15,19 , and 20 [18].

As linkage studies lack power to identify alleles with modest effects or those that interact with other genetic or environmental factors on disease risk, genome-wide association studies (GWASs) using dense sets of SNPs across the genome have rapidly advanced our understanding of the genetic background of complex diseases [19]. The Starr County Health Studies has also reported GWAS results for type 2 diabetes among Mexican-Americans [20] with supportive replications from other studies [21-23]. Utilizing stereoscopic fundus photography within this population, we present here the first genome-wide association analysis of diabetic retinopathy among Mexican-Americans with type 2 diabetes from Starr County, Texas.

\section{Materials and Methods}

2.1. Study Subjects. This study includes 286 individuals with type 2 diabetes representing the earliest onset sibling having fundus photography from sibships with two or more type 2 diabetes-affected siblings from an ongoing genetic study of diabetic retinopathy among Mexican-Americans in Starr County, Texas $[18,20]$. Baseline characteristics were obtained from personal interviews and physical examinations. Fasting blood samples and urine specimens were collected for glycemic, lipid, and microalbuminuria measurements, as previously described [24].

2.2. Genotyping. DNA samples extracted from participants were genotyped using the Affymetrix GeneChip Human Mapping $100 \mathrm{~K}$ Set $[20,25]$. Genotypes were called using the GEL algorithm [26] given its improved call rate and consistent results with other calling methods [20]. According to the annotation file released in March, 2008 from the Affymetrix NetAffx Analysis Center (http://www.affymetrix .com/analysis/index.affx) and the National Center of Biotechnology Information (NCBI) Human Genome Build 36 data, we analyzed 112,666 autosomal SNPs that can be mapped to the NCBI Entrez SNP database and also defined their associated genes in this mapping $100 \mathrm{~K}$ set.

2.3. Diabetic Retinopathy Grading. All participants completed detailed ophthalmologic examinations including stereoscopic color fundus photography of the seven standard fields from the Diabetic Retinopathy Study (DRS) for each eye [27]. Photographs were sent to the University of Wisconsin Reading Center for examination and grading [28] using the Early Treatment Diabetic Retinopathy Study (ETDRS) adaptation of the modified Airlie House classification system [29]. The score for the more severely affected eye of an individual was used to classify retinopathy status. Since it has been shown that familial factors seem to especially influence the severity of diabetic retinopathy $[10,12]$, we focused this analysis on severe diabetic retinopathy and defined our cases and controls as follows. ETDRS grade 10-37: normal to early nonproliferative diabetic retinopathy (NPDR-E) as controls, and 43-85: moderate-to-severe nonproliferative diabetic retinopathy (NPDR-S) and proliferative diabetic retinopathy (PDR) as cases.

2.4. Quality Control and Population Stratification. Fisher's Exact tests of Hardy-Weinberg Equilibrium for controls and for all samples, as well as $\chi^{2}$ tests for the distribution of missing genotypes between cases and controls, were conducted for all 112,666 autosomal SNPs. SNP quality was also assessed based on genotype call rate and minor allele frequency (MAF). A complete-linkage hierarchical clustering method implemented in PLINK [30] was conducted to explore any possible substructure among study subjects using pairwise identity-by-state (IBS) distance across the genomewide SNP data. SNPs with a genotyping rate $<95 \%$, minor allele frequencies $<1 \%$ in all study subjects, and $P$-value of exact Hardy-Weinberg Equilibrium test $<.001$ both in the entire study subjects and control group were tagged for potential quality control issues. A genomic inflation factor ( $\lambda$ ) [31] and mean $\chi^{2}$ statistics generated from all tested SNPs were also calculated to evaluate the effect of population stratification. The quantile-quantile plot of observed and expected distributions of $P$-values was used to assess any distortion of observed distribution from the null.

2.5. Imputation of Untyped SNPs. To expand the genomic coverage, we applied a computationally efficient hidden Markov Chain model [32] programmed in $\mathrm{MACH}$ (http://www.sph.umich.edu/csg/abecasis/MACH/) [33] to impute autosomal genotypes that were present in HapMap Phase III data in the Mexican population from Los Angeles, California, but not genotyped in the Affymetrix $100 \mathrm{~K}$ SNP set. An average allele dosage was estimated with 100 iterations of the imputation algorithm conditional on a set of known haplotypes while simultaneously estimating the recombination map. The squared correlation $\left(r^{2}\right)$ between imputed and true genotypes was estimated for each SNP to evaluate imputation performance [33]. The mask option within MACH was also used to hide $2 \%$ of genotypes from the haplotyping, and imputed genotypes at these locations 
were compared with the actual genotypes to estimate the imputation error rate.

2.6. Statistical Analysis. $\chi^{2}$ tests, Student $T$-tests, and logistic regressions were used to compare the basic characteristics between cases and controls using SAS/STAT system (SAS Institute Inc., Cary, NC). For single-marker case-control analyses, we did not initially filter any SNPs for qualitycontrol reasons, but we labeled them with quality-control indicators for data interpretation. Logistic regression under an additive genetic model was performed for each directly genotyped SNP adjusting for the effects of age, gender, diabetes duration, and serum glycosylated hemoglobin level using PLINK [30]. Imputed markers with poor performance were filtered to obtain more reliable association results based on the per marker quality measures generated from $\mathrm{MACH}$. A cutoff of 0.5 for $r^{2}$ between imputed and true genotypes was applied to remove about $90 \%$ of poorly imputed SNPs at a cost of $5 \%$ of good ones. To account for the probability distribution and the uncertainty of genotype imputation, logistic regression modeling adjusted for the same covariates was performed on these well-imputed markers using ProbABEL (http://mga.bionet.nsc.ru/ yurii/ABEL/) from the ABEL set of programs $[34,35]$, where the SNP effect was assessed by its average imputed allele dosage.

\section{Results}

Among 286 Mexican-Americans with type 2 diabetes from Starr County, Texas, 103 (36\%) with severe nonproliferative or proliferative diabetic retinopathy were defined as cases. Compared to those without retinopathy or only with modest nonproliferative retinopathy, individuals with severe diabetic retinopathy had significantly longer duration of diabetes, higher glycohemoglobin levels, and higher systolic blood pressure measurements (all $P$-values of Student $t$-test $<.005$, Table 1) [36].

The average genotype call rate among study subjects was $93 \%$, with $14 \%$ of the 112,666 autosomal SNPs having a call rate less than $90 \%$, and $4 \%$ having $P$-values for $\chi^{2}$ tests for genotype missingness between cases and controls less than .01. All polymorphic SNPs in this study $(n=111,528)$ remained in the single-marker analysis, and $5641(5 \%)$ of them had minor allele frequency (MAF) less than $1 \%$. The mean $\chi^{2}$ statistics and the genomic inflation factor $(\lambda)$ were 1.02 and 1.06, respectively, suggesting that any observed associations will unlikely be due to population stratification. However, there might be a slightly increased false positive rate since both values are greater than 1 . There was no subpopulation identified from our study subjects using the IBS clustering analysis, and none of the permutation tests of IBS differences between cases and controls were significant (data not shown). The Q-Q plot in Supplementary Figure 1 also shows no strong evidence of population stratification, but with a deviation toward null possibly due to decreased power.

Figure 1 shows the $P$-value distribution for single-marker associations with severe retinopathy under an additive genetic model adjusted for age, gender, diabetes duration, and glycosylated hemoglobin level across the whole genome using the Affymetrix $100 \mathrm{~K}$ SNP set. All the top signals met all quality control criteria. Detailed information for the 10 strongest single marker associations is presented in Table 2. (Results for all SNPs are available as Supplementary Table 1) The best signals found in this study were rs2300782 $(P=$ $\left.6.04 \times 10^{-5}\right)$ in $5 \mathrm{q} 21$ and $\mathrm{rs} 10519765\left(P=6.21 \times 10^{-5}\right)$ in $15 \mathrm{q} 13$, that locate in the intron of the calcium/calmodulindependent protein kinase IV (CAMK4) gene and formin 1 (FMN1) gene, respectively.

The $100 \mathrm{~K} \mathrm{SNP}$ set alone is insufficient to cover most variants currently available, therefore, we extended the analysis to an imputation-based GWAS using HapMap data [37]. To improve imputation performance, one subject with an overall genotyping rate less than $90 \%$ and 8,094 SNPs not meeting quality control criteria were excluded from imputation. A total of $1,326,990$ SNPs from the HapMap Phase III Mexican population in Los Angeles, California, were imputed on 102 cases and 183 controls of severe diabetic retinopathy based on 104,572 directly genotyped SNPs. Supplementary Table 2 summarizes the imputation performance of MACH [33] across the whole genome. More than $88 \%$ of the imputed SNPs had a minor allele frequency greater than $1 \%$, and the $\mathrm{MACH}$ estimated per allele error rate after masking $2 \%$ of direct genotypes from imputation was $8.7 \%$. For all imputed markers, the overall $r^{2}$ between imputed and true genotypes was 0.387 , and the average posterior probability for the most likely genotypes was 0.783 . The 421,010 (31.7\%) markers considered well imputed with an $r^{2} \geq 0.5$ were used for subsequent association analysis.

Figure 2 shows the genome-wide $P$-value distribution from the logistic regression models using the well-imputed SNPs. The genomic inflation factor $(\lambda)$ and the mean $\chi^{2}$ statistics were 1.03 and 0.47 , respectively, implying that any observed associations are unlikely due to population stratification. Table 3 summarizes the genes nearest the top signals $\left(P\right.$-value less than $\left.10^{-4}\right)$ under logistic regression models adjusted for the effects of age, gender, diabetes duration, and glycosylated hemoglobin level. There were 32 SNPs associated with severe diabetic retinopathy at $P$-value less than $10^{-4}$, with 7 of these top signals in chromosomal region $6 \mathrm{p} 11-12$, where tubulointerstitial nephritis antigen (TINAG) is encoded.

\section{Discussion}

Genome-wide association studies provide an additional tool, in conjunction with candidate gene and linkage studies to better understand the genetics of diabetic retinopathy. Our strongest signals from single-marker analysis implicated several genes as associated with severe diabetic retinopathy. SNP rs2300782 is located in an intronic region of CAMK4; its product has been reported within the $\mathrm{Ca}(2+) /$ calmodulindependent protein kinase subfamily and has been shown to increase transcriptional activity required for ATF-2 induced insulin gene expression [38]. The second best single-marker signal rs10519765 is located in FMN1, encoding a protein 


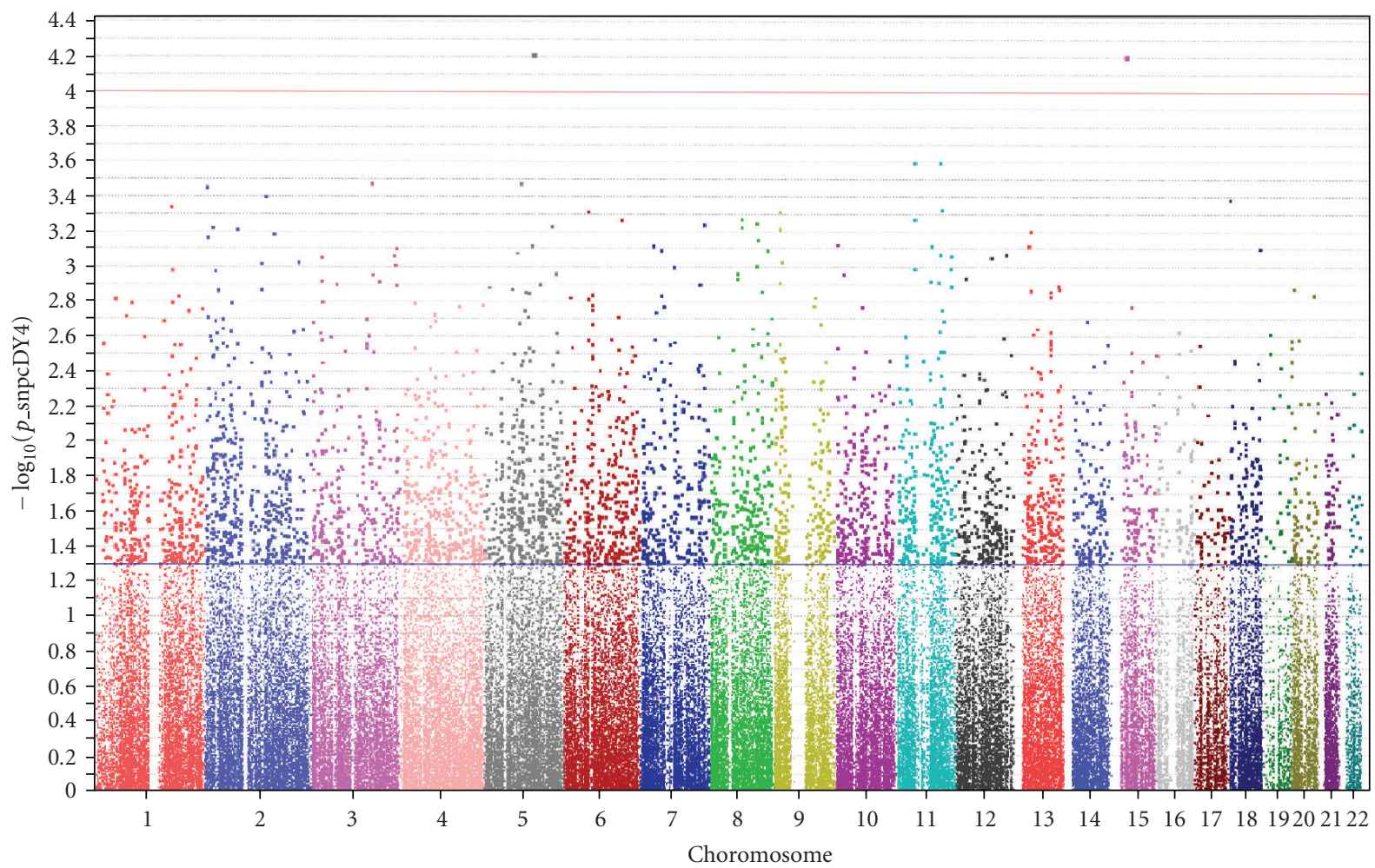

FIGURE 1: Summary of genome-wide associations between 111,528 SNPs and severe diabetic retinopathy under additive genetic model adjusted for age, gender, diabetes duration, and HbAlc level.

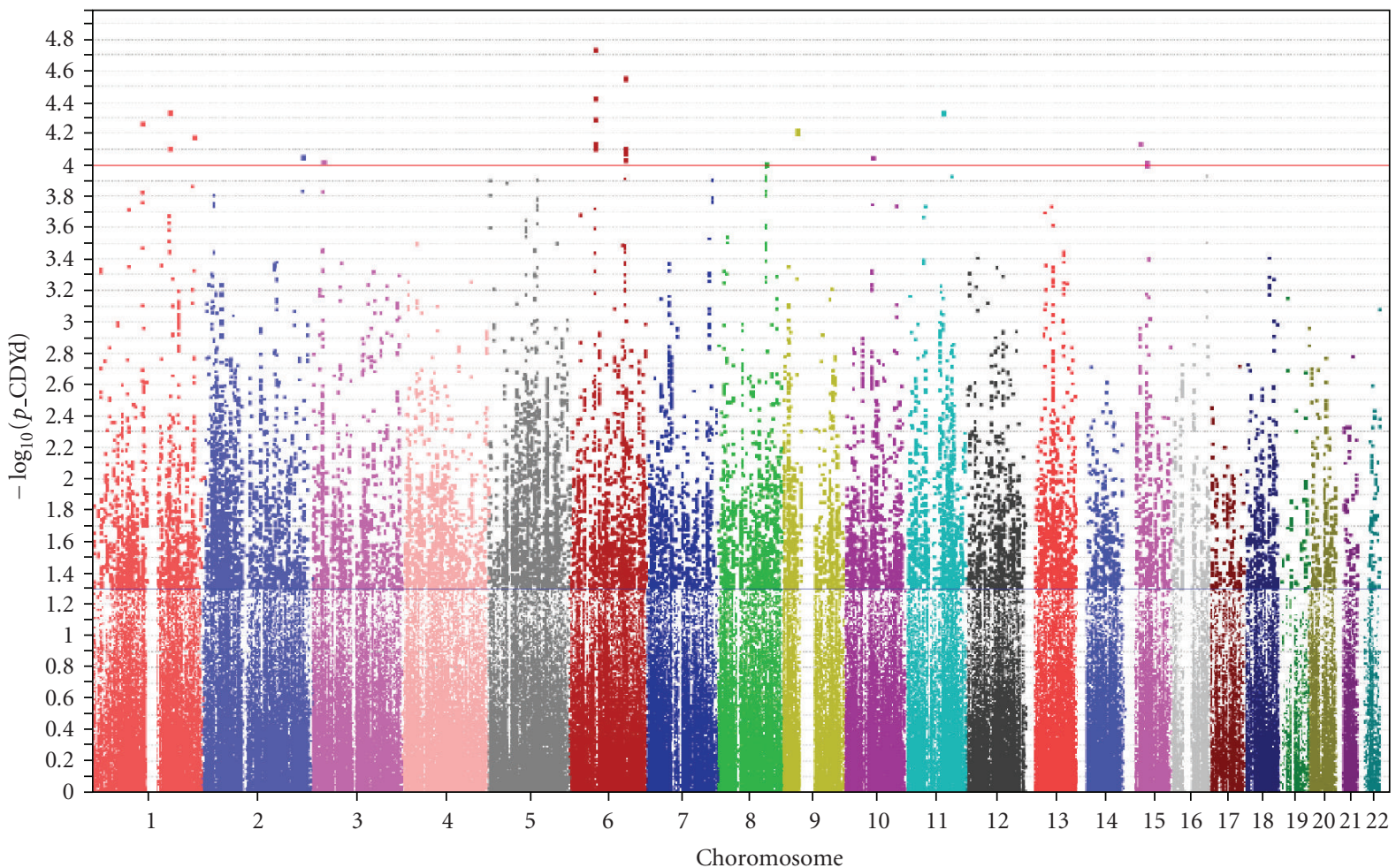

FIGURE 2: Summary of genome-wide associations between 421,010 well-imputed SNPs and severe diabetic retinopathy under additive genetic model adjusted for age, gender, diabetes duration, and HbA1c level. 
TABle 1: Basic characteristics of Mexican-Americans with type 2 diabetes from Starr County, Texas.

\begin{tabular}{|c|c|c|c|c|c|c|}
\hline Variables & & $\begin{array}{c}\text { No DR + NPDR-E } \\
(\text { controls } n=183) \\
\end{array}$ & $\begin{array}{l}\text { NPDR-S + PDR }{ }^{\#} \\
(\text { cases } n=103)\end{array}$ & $P$-value ${ }^{\S}$ & OR & $(95 \% \mathrm{CI})$ \\
\hline Age & Mean \pm SD & $57.06 \pm 11.54$ & $58.67 \pm 9.27$ & .1988 & 1.01 & $(0.99-1.04)$ \\
\hline \multirow[t]{2}{*}{ Gender } & Male & $66(36.07 \%)$ & $43(41.75 \%)$ & .3422 & 1.00 & - \\
\hline & Female & $117(63.93 \%)$ & $60(58.25 \%)$ & & 0.79 & $(0.48-1.29)$ \\
\hline \multirow[t]{4}{*}{ Albumin } & Negative & $132(74.58 \%)$ & $57(60.64 \%)$ & .0802 & 1.00 & - \\
\hline & $20 \mathrm{mg} / \mathrm{L}$ & $22(12.43 \%)$ & $14(14.89 \%)$ & & 1.47 & $(0.70-3.08)$ \\
\hline & $50 \mathrm{mg} / \mathrm{L}$ & $17(9.60 \%)$ & $17(18.09 \%)$ & & 2.32 & $(1.10-4.86)^{*}$ \\
\hline & $100 \mathrm{mg} / \mathrm{L}$ & $6(3.39 \%)$ & $6(6.38 \%)$ & & 2.32 & $(0.72-7.49)$ \\
\hline \multirow[t]{2}{*}{ Hypertension history } & No & $96(54.24 \%)$ & $59(59.60 \%)$ & .3895 & 1.00 & - \\
\hline & Yes & $81(45.76 \%)$ & $40(40.40 \%)$ & & 0.80 & $(0.49-1.32)$ \\
\hline Diabetes duration (yrs) & mean $\pm \mathrm{SD}$ & $12.02 \pm 9.09$ & $18.10 \pm 8.22$ & $<.0001$ & 1.08 & $(1.05-1.11)^{* * *}$ \\
\hline $\operatorname{HbA1C}(\%)$ & mean $\pm \mathrm{SD}$ & $10.87 \pm 3.72$ & $12.29 \pm 3.90$ & .0028 & 1.10 & $(1.03-1.18)^{* * *}$ \\
\hline Fasting glucose (mg/dL) & mean $\pm S D$ & $185.54 \pm 68.71$ & $201.63 \pm 84.49$ & .0883 & 1.03 & $(0.99-1.06)$ \\
\hline $\mathrm{BMI}\left(\mathrm{kg} / \mathrm{m}^{2}\right)$ & mean $\pm \mathrm{SD}$ & $31.82 \pm 6.49$ & $30.85 \pm 5.93$ & .2175 & 0.98 & $(0.94-1.02)$ \\
\hline Systolic BP (mmHg) & mean $\pm \mathrm{SD}$ & $126.79 \pm 17.86$ & $134.73 \pm 23.26$ & .0037 & 1.21 & $\begin{array}{l}(1.07-1.38)^{* * *} \\
\quad(\text { every } 10 \text { unit })\end{array}$ \\
\hline Diastolic BP (mmHg) & mean $\pm S D$ & $72.69 \pm 10.03$ & $73.32 \pm 10.78$ & .6290 & 1.06 & $\begin{array}{l}(0.84-1.35) \\
\quad(\text { every } 10 \text { unit })\end{array}$ \\
\hline Total cholesterol (mg/dL) & mean $\pm S D$ & $191.21 \pm 41.81$ & $199.06 \pm 42.13$ & .1381 & 1.05 & $\begin{array}{l}\text { (0.99-1.11) } \\
\quad \text { (every } 10 \text { unit })\end{array}$ \\
\hline Triglycerides (mg/dL) & mean $\pm S D$ & $184.79 \pm 120.92$ & $210.02 \pm 123.59$ & .1031 & 1.02 & $\begin{array}{l}\text { (0.99-1.04) } \\
\quad \text { (every } 10 \text { unit) }\end{array}$ \\
\hline HDL cholesterol (mg/dL) & mean $\pm \mathrm{SD}$ & $42.86 \pm 11.73$ & $40.62 \pm 10.32$ & .1153 & 0.83 & $\begin{array}{l}(0.66-1.05) \\
\quad(\text { every } 10 \text { unit })\end{array}$ \\
\hline LDL cholesterol (mg/dL) & mean $\pm \mathrm{SD}$ & $111.49 \pm 30.71$ & $118.94 \pm 34.84$ & .0790 & 1.08 & $(0.99-1.16)$ \\
\hline
\end{tabular}

\#No DR: normal or nondiabetic retinopathy; NPDR-E: early nonproliferative diabetic retinopathy

\#NPDR-S: moderate-to-severe nonproliferative diabetic retinopathy; PDR: proliferative diabetic retinopathy

$\S_{P}$-value of $\chi^{2}$ test or Student $t$-test

${ }^{*} P$-value $<.05,{ }^{* *} P$-value $<.01,{ }^{* * *} P$-value $<.005$.

involved in cell adhesion and morphogenesis by assembling radial action cables in epithelial cells $[39,40]$. However, there is very limited evidence about genetic variation on CAMK4 or FMN1 and diabetes or its complications.

CNTN5 (contactin 5) at 11q22 is also among the nominally associated genes for severe diabetic retinopathy. The protein encoded by CNTN5 belongs to an immunoglobulin superfamily and may participate in the developing nervous system [41]. SNP variants in CNTN5 have been reported to be associated with atrial fibrillation and heart failure [42]. Another top single-marker signal was in COLEC12 (collectin subfamily member 12), encoding a protein of the C-lectin family, that possesses collagen-like sequences and carbohydrate recognition domains. This protein is a scavenger receptor recognizing oxidized phospholipids, so it may participate in removing oxidative damage [43], which is a potential etiological factor for diabetic retinopathy [44]. API5, EDIL3, BFSP2, HNMT, and SCYL1BP1 were also among the top signals from the single-marker analysis within their associated region, but there is no clear connection currently between these genes and diabetes or its complications.

Imputation of untyped SNP markers is as a useful tool to improve the coverage and power for genome-wide association studies without additional genotyping costs [45, 46]. A number of programs have been developed and routinely used to impute genotypes based on the observed haplotype structure from millions of SNPs in the HapMap project [37] or 1000 Genomes Project [47]. Given the uneven coverage of the genome by the Affymertrix $100 \mathrm{~K} \mathrm{SNP}$ set, a more detailed SNP map was obtained by imputation conducted in $\mathrm{MACH}$ [32], since it consistently outperformed other algorithms with better accuracy and efficiency $[48,49]$. The Mexican-American population in Starr County is relatively homogenous with $97.5 \%$ self-reporting as Hispanic. Genetically, this population is admixed with $68 \%$ European, $27 \%$ Asian, and 6\% African ancestry [20]. Considering the importance of linkage disequilibrium pattern between the reference panel and study subjects, we used the HapMap III population with Mexican ancestry from Los Angles, California, who identified themselves as having at least three grandparents born in Mexico, as the reference set for imputation. The overall $8.7 \%$ estimate from $\mathrm{MACH}$ of overall allele error rate was comparable with the original $\mathrm{MACH}$ evaluation data (7.5\%) [32]. Compared to all the autosomal SNP variants $(n=1,387,466)$ available in the HapMap III Mexican population, the genomic coverage in 
TABLE 2: Top 10 single-marker associations with severe diabetic retinopathy among Mexican-Americans from Starr County, Texas.

\begin{tabular}{|c|c|c|c|c|c|c|c|c|c|}
\hline dbSNP rs\# & Chr & Position* & $\begin{array}{l}\text { Minor/major } \\
\text { allele }\end{array}$ & $\begin{array}{l}\text { MAF in } \\
\text { Cases }\end{array}$ & $\begin{array}{l}\text { MAF in } \\
\text { Controls }\end{array}$ & $P$-value ${ }^{\S}$ & $\begin{array}{c}\mathrm{OR}^{+} \\
(95 \% \mathrm{CI})\end{array}$ & Nearest gene* & $\begin{array}{l}\text { Distance/ } \\
\text { relationship to } \\
\text { gene }\end{array}$ \\
\hline rs 2300782 & 5 & 110816684 & $\mathrm{~A} / \mathrm{G}$ & 0.512 & 0.322 & $6.04 E-05$ & $\begin{array}{c}2.64 \\
(1.64-4.25)\end{array}$ & CAMK4 & intron/0 \\
\hline rs10519765 & 15 & 30992716 & $\mathrm{~A} / \mathrm{G}$ & 0.136 & 0.294 & $6.21 E-05$ & $\begin{array}{c}0.30 \\
(0.16-0.54)\end{array}$ & FMN1 & intron/0 \\
\hline rs899036 & 11 & 41639486 & $\mathrm{C} / \mathrm{A}$ & 0.125 & 0.210 & $2.52 E-04$ & $\begin{array}{c}0.32 \\
(0.17-0.59)\end{array}$ & API5 & $\begin{array}{l}\text { upstream/ } \\
1650623\end{array}$ \\
\hline rs 10501943 & 11 & 99452209 & $\mathrm{C} / \mathrm{T}$ & 0.195 & 0.086 & $2.53 E-04$ & $\begin{array}{c}3.04 \\
(1.68-5.52)\end{array}$ & CNTN5 & intron/0 \\
\hline rs1445754 & 5 & 83611387 & $\mathrm{~A} / \mathrm{T}$ & 0.143 & 0.273 & $3.35 E-04$ & $\begin{array}{c}0.37 \\
(0.22-0.64)\end{array}$ & EDIL3 & intron/0 \\
\hline rs1197310 & 3 & 134610914 & $\mathrm{~T} / \mathrm{A}$ & 0.540 & 0.435 & $3.35 E-04$ & $\begin{array}{c}2.25 \\
(1.45-3.51)\end{array}$ & BFSP2 & intron/0 \\
\hline rs699549 & 2 & 4683138 & $\mathrm{~T} / \mathrm{C}$ & 0.115 & 0.043 & $3.49 E-04$ & $\begin{array}{c}4.27 \\
(1.93-9.47)\end{array}$ & - & - \\
\hline rs763970 & 2 & 138352603 & $\mathrm{~A} / \mathrm{C}$ & 0.330 & 0.206 & $4.00 E-04$ & $\begin{array}{c}2.25 \\
(1.44-3.52)\end{array}$ & HNMT & $\begin{array}{l}\text { upstream/ } \\
85675\end{array}$ \\
\hline rs599019 & 18 & 284495 & $\mathrm{G} / \mathrm{T}$ & 0.030 & 0.131 & $4.06 E-04$ & $\begin{array}{c}0.15 \\
(0.05-0.43)\end{array}$ & COLEC12 & $\begin{array}{l}\text { downstream/ } \\
24861\end{array}$ \\
\hline rs6427247 & 1 & 168647104 & G/A & 0.360 & 0.221 & $4.56 E-04$ & $\begin{array}{c}2.17 \\
(1.41-3.35)\end{array}$ & SCYL1BP1 & $\begin{array}{l}\text { upstream/ } \\
120790\end{array}$ \\
\hline
\end{tabular}

*Affymetrix NetAffx annotation build 25, NCBI genome build 36.1.

$\S P$-value of logistic regression under additive genetic model, adjusted for age, gender, diabetes duration, and HbAlc level.

${ }^{+}$odds ratios of the minor allele.

TABLE 3: Regions with strongest imputation-inferred associations $(P<.0001)$ using estimated allelic dosage under an adjusted additive genetic model.

\begin{tabular}{|c|c|c|c|c|c|}
\hline Location & $\begin{array}{l}\text { SNP with best } P \text {-value } \\
\text { within this region }\end{array}$ & Position* & Best $P$-value ${ }^{\S}$ & $\begin{array}{c}\text { \# of top SNPs } \\
\text { within this region }\end{array}$ & Nearest gene* \\
\hline $6 \mathrm{p} 11-12$ & rs6909083 & 54290262 & $1.80 E-05$ & 7 & TINAG \\
\hline $6 q 22$ & rs17083119 & 121443809 & $2.76 E-05$ & 4 & C6orf170 \\
\hline $1 \mathrm{q} 23$ & rs1033465 & 171254353 & $4.50 E-05$ & 2 & TNFSF18 \\
\hline $1 \mathrm{p} 13$ & rs11583330 & 109925036 & $5.35 E-05$ & 1 & GNAI3 \\
\hline $1 q 42$ & rs3014267 & 225619540 & $6.58 E-05$ & 1 & CDC42BPA \\
\hline $15 q 13$ & rs11635920 & 30999949 & $7.18 E-05$ & 4 & FMN1, GREM1 \\
\hline $2 q 35-36$ & rs6726798 & 219009099 & $8.66 E-05$ & 1 & VIL1 \\
\hline $10 \mathrm{q} 21$ & rs11812882 & 59712631 & $8.85 E-05$ & 1 & ZCD1 \\
\hline $2 \mathrm{q} 35$ & rs1106412 & 219023301 & $8.91 E-05$ & 1 & USP37 \\
\hline $3 \mathrm{p} 24$ & rs11927173 & 23200198 & $9.39 E-05$ & 2 & UBE2E2 \\
\hline $8 \mathrm{q} 22$ & rs3098241 & 104494480 & $9.72 E-05$ & 2 & SLC25A32 \\
\hline
\end{tabular}

*Affymetrix NetAffx annotation build 25, NCBI genome build 36.1

$\S P$-value of logistic regression under additive genetic model, adjusted for age, gender, diabetes duration, and HbA1C level.

the imputation analysis increases extensively with $30.3 \%$ $(n=421,010)$ of the variants tested here, whereas only $4.3 \%$ $(n=60,283)$ of them are directly covered in the Affymetrix $100 \mathrm{~K}$ SNP set.

Among all imputed markers associated with severe diabetic retinopathy at a $P$-value less than $10^{-4}$, there are 7 SNPs located in 6p11-12 where TINAG (tubulointerstitial nephritis antigen) is encoded. TINAG is a glycoprotein originally identified as a target antigen involved in human antitubular basement membrane disease [50]. It has been recognized as an extracellular matrix protein and shows increased expression in the kidney of streptozotocin-diabetic rats [51], but there is yet no evidence how TINAG might be involved in human diabetes or its complications. Chromosomal region 15q13, where FMN1 (formin 1) and GREM1 (gremlin 1) map, also has 4 of the strongest imputationbased signals. FMN1 has one of the strongest associations from direct genotyping (rs10519765). Gremlin is located 
$\sim 40 \mathrm{~kb}$ downstream of FMN1, a highly conserved protein involved in various disorders related to fibrotic changes in the kidney, lung, and liver [52]. This protein has been shown to have a potential role in diabetic nephropathy because of enhanced expression and interaction with TGF- $\beta$ signaling pathways [53, 54]. Recent evidence of increased gremlin mRNA in bovine retinal perictyes [55] and immunohistochemistry data on the mouse retina [54] coupled with results reported here elevate interest in gremlin.

In addition to identifying novel candidate genes, we were interested in SNPs among genes that appear to be traditional candidates for diabetic retinopathy. A list of 208 retinopathy candidate genes has been generated [18, 56] (see Supplementary Table 3) to include genes involved in metabolic processes or clinical risk factors leading to retinopathy and those that have been previously reported to be associated with diabetic retinopathy. Among a total of 667 SNPs that are located in the coding region of these 208 candidate genes and also genotyped in the Affymetrix $100 \mathrm{~K}$ SNP set, 54 of them had nominal allelic association with diabetic retinopathy with $P$-values ranging from .001 to .05 (Supplementary Figure 2). The significance pattern found here is more than expected by chance alone.

Although we cannot completely exclude the possibility of undetected subpopulations affecting the results, we found no evidence of population stratification. Hayes et al. also used the genome-wide SNP data to estimate proportions of ancestry on the same population. Ancestry is unlikely to be a source of spurious association in this sample since the amount of African, Asian, and European ancestries were indistinguishable between cases and controls [20].

The primary objective of this study is searching for diabetic retinopathy genes without any assumption about the location of disease-associating variants. This initial genomewide association analysis of severe diabetic retinopathy identified several unexpected loci that may contribute to the genetic susceptibility of diabetic retinopathy. The study was limited, however, in its ability to detect small effect sizes. A sample of 103 cases and 183 controls was only powerful enough to identify variants with odds ratios greater than 2.2 (assuming disease allele frequency of 0.5 and disease prevalence of 0.5 in an additive model, calculated by CaTS [57]) with a genome-wide significance level $(P$ value less than $\left.5 \times 10^{-8}\right)$. On the other hand, genetic association studies usually require replication in appropriate independent samples to validate any findings. No such comparable data among Mexican-Americans are available though we are expanding the sample and SNP coverage, even so, these results set some limits as to the magnitude of genetic effects for diabetic retinopathy.

In summary, we observed several SNPs and genes associated with severe diabetic retinopathy in this initial genomewide analysis. None of these loci have been previously linked to diabetic retinopathy or diabetes itself. While the underlying mechanisms leading to diabetic retinopathy remain unresolved, these results implicate genetic regulation of oxidative stress and cell adhesion as possible players in the development of diabetic retinopathy.

\section{References}

[1] A. Foster and S. Resnikoff, "The impact of Vision 2020 on global blindness," Eye, vol. 19, no. 10, pp. 1133-1135, 2005.

[2] Centers for Disease Control and Prevention, National Diabetes Fact Sheet: General Information and National Estimates on Diabetes in the United States, 2005, Department of Health and Human Services, Centers for Disease Control and Prevention, Atlanta, Ga, USA, 2005.

[3] J. B. Saaddine, A. A. Honeycutt, K. M. V. Narayan, X. Zhang, R. Klein, and J. P. Boyle, "Projection of diabetic retinopathy and other major eye diseases among people with diabetes mellitus: United States, 2005-2050," Archives of Ophthalmology, vol. 126, no. 12, pp. 1740-1747, 2008.

[4] H. C. Looker, J. Krakoff, W. C. Knowler, P. H. Bennett, R. Klein, and R. L. Hanson, "Longitudinal studies of incidence and progression of diabetic retinopathy assessed by retinal photography in Pima Indians," Diabetes Care, vol. 26, no. 2, pp. 320-326, 2003.

[5] M. C. Leske, S.-Y. Wu, A. Hennis et al., "Hyperglycemia, blood pressure, and the 9-year incidence of diabetic retinopathy: the Barbados Eye Studies," Ophthalmology, vol. 112, no. 5, pp. 799-805, 2005.

[6] L. Cikamatana, P. Mitchell, E. Rochtchina, S. Foran, and J. J. Wang, "Five-year incidence and progression of diabetic retinopathy in a defined older population: the Blue Mountains Eye Study," Eye, vol. 21, no. 4, pp. 465-471, 2007.

[7] R. Singh, K. Ramasamy, C. Abraham, V. Gupta, and A. Gupta, "Diabetic retinopathy: an update," Indian Journal of Ophthalmology, vol. 56, no. 3, pp. 179-188, 2008.

[8] M. I. Harris, R. Klein, C. C. Cowie, M. Rowland, and D. D. Byrd-Holt, "Is the risk of diabetic retinopathy greater in nonHispanic blacks and Mexican Americans than in non-Hispanic whites with type 2 diabetes? A U.S. population study," Diabetes Care, vol. 21, no. 8, pp. 1230-1235, 1998.

[9] N. Emanuele, J. Sacks, R. Klein et al., "Ethnicity, race, and baseline retinopathy correlates in the Veterans Affairs Diabetes Trial," Diabetes Care, vol. 28, no. 8, pp. 1954-1958, 2005.

[10] "Clustering of long-term complications in families with diabetes in the diabetes control and complications trial. The Diabetes Control and Complications Trial Research Group," Diabetes, vol. 46, no. 11, pp. 1829-1839, 1997.

[11] M. Rema, G. Saravanan, R. Deepa, and V. Mohan, "Familial clustering of diabetic retinopathy in South Indian Type 2 diabetic patients," Diabetic Medicine, vol. 19, no. 11, pp. 910916, 2002.

[12] D. M. Hallman, J. C. Huber Jr., V. H. Gonzalez, B. E. K. Klein, R. Klein, and C. L. Hanis, "Familial aggregation of severity of diabetic retinopathy in Mexican Americans from Starr County, Texas," Diabetes Care, vol. 28, no. 5, pp. 11631168, 2005.

[13] N. H. Arar, B. I. Freedman, S. G. Adler et al., "Heritability of the severity of diabetic retinopathy: the FIND-Eye study," Investigative Ophthalmology and Visual Science, vol. 49, no. 9, pp. 3839-3845, 2008.

[14] K. M. Warpeha and U. Chakravarthy, "Molecular genetics of microvascular disease in diabetic retinopathy," Eye, vol. 17, no. 3, pp. 305-311, 2003.

[15] K. Uhlmann, P. Kovacs, Y. Boettcher, H.-P. Hammes, and R. Paschke, "Genetics of diabetic retinopathy," Experimental and Clinical Endocrinology and Diabetes, vol. 114, no. 6, pp. 275$294,2006$. 
[16] G. Imperatore, R. L. Hanson, D. J. Pettitt, S. Kobes, P. H. Bennett, and W. C. Knowler, "Sib-pair linkage analysis for susceptibility genes for microvascular complications among Pima Indians with 2 diabetes," Diabetes, vol. 47, no. 5, pp. 821830, 1998.

[17] H. C. Looker, R. G. Nelson, E. Chew et al., "Genome-wide linkage analyses to identify loci for diabetic retinopathy," Diabetes, vol. 56, no. 4, pp. 1160-1166, 2007.

[18] D. M. Hallman, E. Boerwinkle, V. H. Gonzalez, B. E. K. Klein, R. Klein, and C. L. Hanis, "A genome-wide linkage scan for diabetic retinopathy susceptibility genes in Mexican Americans with type 2 diabetes from Starr County, Texas," Diabetes, vol. 56, no. 4, pp. 1167-1173, 2007.

[19] "A catalog of published genome-wide association studies," http://www.genome.gov/26525384.

[20] M. G. Hayes, A. Pluzhnikov, K. Miyake et al., "Identification of type 2 diabetes genes in Mexican Americans through genomewide association studies," Diabetes, vol. 56, no. 12, pp. 30333044, 2007.

[21] R. L. Hanson, C. Bogardus, D. Duggan et al., "A search for variants associated with young-onset type 2 diabetes in American Indians in a 100K genotyping array," Diabetes, vol. 56, no. 12, pp. 3045-3052, 2007.

[22] E. Rampersaud, C. M. Damcott, M. Fu et al., "Identification of novel candidate genes for type 2 diabetes from a genomewide association scan in the old order amish: evidence for replication from diabetes-related quantitative traits and from independent populations," Diabetes, vol. 56, no. 12, pp. 3053 3062, 2007.

[23] J. C. Florez, A. K. Manning, J. Dupuis et al., "A 100K genomewide association scan for diabetes and related traits in the Framingham Heart Study: replication and integration with other genome-wide datasets," Diabetes, vol. 56, no. 12, pp. 3063-3074, 2007.

[24] E. Boerwinkle, C. A. Brown, M. Carrejo et al., "Multi-center genetic study of hypertension: the Family blood pressure program (FBPP)," Hypertension, vol. 39, no. 1, pp. 3-9, 2002.

[25] H. Matsuzaki, S. Dong, H. Loi et al., "Genotyping over 100,000 SNPs on a pair of oligonucleotide arrays," Nat Methods, vol. 1, no. 2, pp. 109-111, 2004.

[26] D. L. Nicolae, X. Wu, K. Miyake, and N. J. Cox, "GEL: a novel genotype calling algorithm using empirical likelihood," Bioinformatics, vol. 22, no. 16, pp. 1942-1947, 2006.

[27] "Diabetic retinopathy study. Report number 6. Design, methods, and baseline results. Report number 7. A modification of the airlie house classification of diabetic retinopathy. prepared by the diabetic retinopathy," Investigative Ophthalmology and Visual Science, vol. 21, no. 1, part 2, pp. 1-226, 1981.

[28] R. Klein, B. E. K. Klein, and Y. L. Magli, "An alternative method of grading diabetic retinopathy," Ophthalmology, vol. 93, no. 9, pp. 1183-1187, 1986.

[29] "Grading diabetic retinopathy from stereoscopic color fundus photographs - an extension of the Modified Airlie House classification. ETDRS report number 10. Early Treatment Diabetic Retinopathy Study research group," Ophthalmology, vol. 98, supplement 5, pp. 786-806, 1991.

[30] S. Purcell, B. Neale, K. Todd-Brown et al., "PLINK: a tool set for whole-genome association and population-based linkage analyses," American Journal of Human Genetics, vol. 81, no. 3, pp. 559-575, 2007.

[31] B. Devlin and K. Roeder, "Genomic control for association studies," Biometrics, vol. 55, no. 4, pp. 997-1004, 1999.
[32] Y. Li, C. J. Willer, J. Ding, P. Scheet, and G. R. Abecasis, "Rapid markov chain haplotyping and genotype inference," submitted.

[33] Y. Li and G. R. Abecasis, "Mach 1.0: rapid haplotype reconstruction and missing genotype inference," American Journal of Human Genetics, vol. S79, p. 2290, 2006.

[34] Y. S. Aulchenko, S. Ripke, A. Isaacs, and C. M. van Duijn, "GenABEL: an R library for genome-wide association analysis," Bioinformatics, vol. 23, no. 10, pp. 1294-1296, 2007.

[35] Y. S. Aulchenko, M. V. Struchalin, and C. M. van Duijn, "ProbABEL package for genome-wide association analysis of imputed data," BMC Bioinformatics, vol. 11, article 134, 2010.

[36] L. B. Piller, Y. P. Fu, V. H. Gonzalez, B. E. K. Klein, R. Klein, and C. L. Hanis, Prevalence and incidence of diabetic retinopathy of Mexican-Americans with type 2 diabetes from Starr county, Texas.

[37] K. A. Frazer, D. G. Ballinger, D. R. Cox et al., "A second generation human haplotype map of over 3.1 million SNPs," Nature, vol. 449, no. 7164, pp. 851-861, 2007.

[38] N. Ban, Y. Yamada, Y. Someya et al., "Activating transcripton factor-2 is a positive regulator in CaM kinase IV-induced human insulin gene expression," Diabetes, vol. 49, no. 7, pp. 1142-1148, 2000.

[39] A. Kobielak, H. A. Pasolli, and E. Fuchs, "Mammalian formin1 participates in adherens junctions and polymerization of linear actin cables," Nature Cell Biology, vol. 6, no. 1, pp. 21-30, 2004.

[40] B. L. Goode and M. J. Eck, "Mechanism and function of formins in the control of actin assembly," Annual Review of Biochemistry, vol. 76, pp. 593-627, 2007.

[41] Y. Kamei, Y. Takeda, K. Teramoto, O. Tsutsumi, Y. Taketani, and K. Watanabe, "Human NB-2 of the contactin subgroup molecules: chromosomal localization of the gene (CNTN5) and distinct expression pattern from other subgroup members," Genomics, vol. 69, no. 1, pp. 113-119, 2000.

[42] M. G. Larson, L. D. Atwood, E. J. Benjamin et al., "Framingham Heart Study 100K project: genome-wide associations for cardiovascular disease outcomes," BMC Medical Genetics, vol. 8, supplement 1 , article S5, 2007.

[43] K. Ohtani, Y. Suzuki, S. Eda et al., "The membrane-type collectin CL-P1 is a scavenger receptor on vascular endothelial cells," Journal of Biological Chemistry, vol. 276, no. 47, pp. 44222-44228, 2001.

[44] J. Cai and M. Boulton, "The pathogenesis of diabetic retinopathy: old concepts and new questions," Eye, vol. 16, no. 3, pp. 242-260, 2002.

[45] J. Marchini, B. Howie, S. Myers, G. McVean, and P. Donnelly, "A new multipoint method for genome-wide association studies by imputation of genotypes," Nature Genetics, vol. 39, no. 7, pp. 906-913, 2007.

[46] S. R. Browning, "Missing data imputation and haplotype phase inference for genome-wide association studies," Human Genetics, vol. 124, no. 5, pp. 439-450, 2008.

[47] M. Via, C. Gignoux, and E. G. Burchard, "The 1000 genomes project: new opportunities for research and social challenges," Genome Medicine, vol. 2, no. 1, p. 3, 2010.

[48] M. Nothnagel, D. Ellinghaus, S. Schreiber, M. Krawczak, and A. Franke, "A comprehensive evaluation of SNP genotype imputation," Human Genetics, vol. 125, no. 2, pp. 163-171, 2009.

[49] Y.-F. Pei, J. Li, L. Zhang, C. J. Papasian, and H.-W. Deng, "Analyses and comparison of accuracy of different genotype imputation methods," PLoS ONE, vol. 3, no. 10, Article ID e3551, 2008. 
[50] K. Yoshioka, T. Takemura, and S. Hattori, “Tubulointerstitial nephritis antigen: primary structure, expression and role in health and disease," Nephron, vol. 90, no. 1, pp. 1-7, 2002.

[51] D. Gong, X. Chen, M. Middleditch et al., "Quantitative proteomic profiling identifies new renal targets of copper(II)selective chelation in the reversal of diabetic nephropathy in rats," Proteomics, vol. 9, no. 18, pp. 4309-4320, 2009.

[52] R. J. Wordinger, G. Zode, and A. F. Clark, "Focus on Molecules: gremlin,” Experimental Eye Research, vol. 87, no. 2, pp. 78-79, 2008.

[53] D. W. P. Lappin, C. Hensey, R. McMahon, C. Godson, and H. R. Brady, "Gremlins, glomeruli and diabetic nephropathy," Current Opinion in Nephrology and Hypertension, vol. 9, no. 5, pp. 469-472, 2000.

[54] V. Dolan, M. Murphy, P. Alarcon, H. R. Brady, and C. Hensey, "Gremlin-a putative pathogenic player in progressive renal disease," Expert Opinion on Therapeutic Targets, vol. 7, no. 4, pp. 523-526, 2003.

[55] R. Kane, L. Stevenson, C. Godson, A. W. Stitt, and C. O'Brien, "Gremlin gene expression in bovine retinal pericytes exposed to elevated glucose," British Journal of Ophthalmology, vol. 89, no. 12, pp. 1638-1642, 2005.

[56] M. S. Roy, D. M. Hallman, Y.-P. Fu, M. Machado, and C. L. Hanis, "Assessment of 193 candidate genes for retinopathy in African Americans with type 1 diabetes," Archives of Ophthalmology, vol. 127, no. 5, pp. 605-612, 2009.

[57] A. D. Skol, L. J. Scott, G. R. Abecasis, and M. Boehnke, "Joint analysis is more efficient than replication-based analysis for two-stage genome-wide association studies," Nature Genetics, vol. 38, no. 2, pp. 209-213, 2006. 


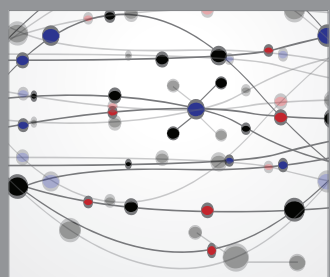

The Scientific World Journal
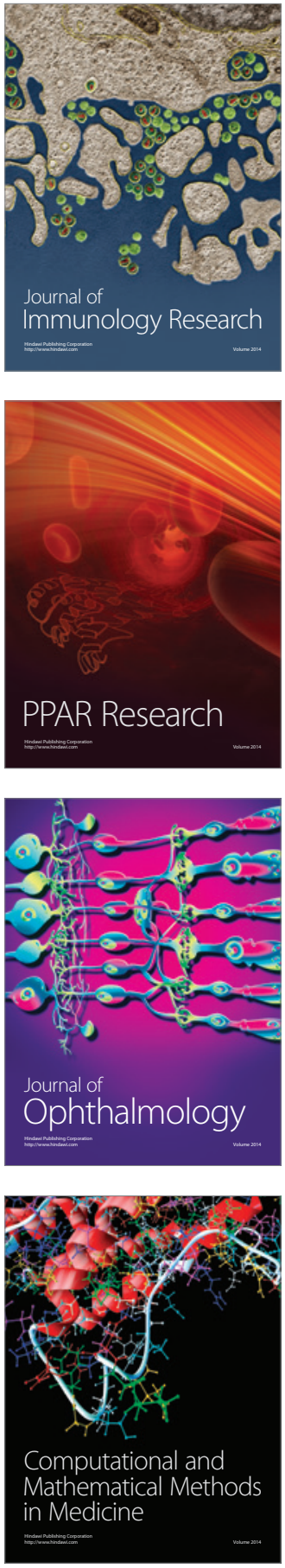

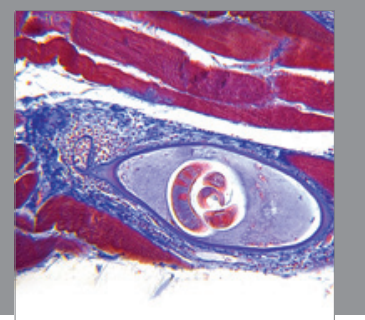

Gastroenterology

Research and Practice
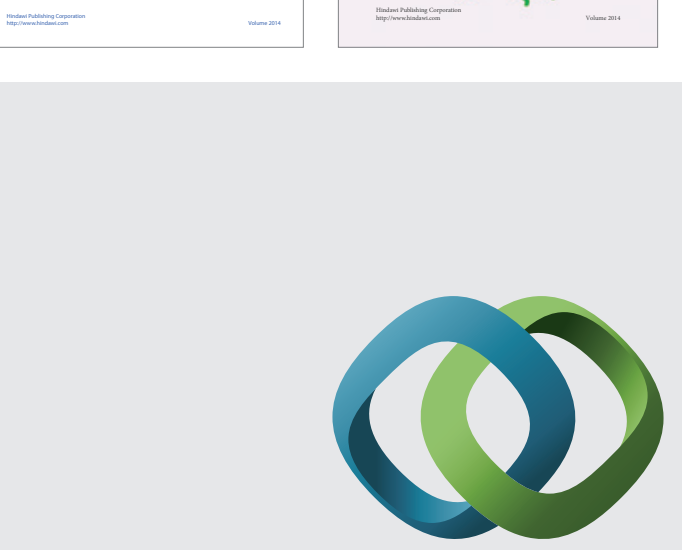

\section{Hindawi}

Submit your manuscripts at

http://www.hindawi.com
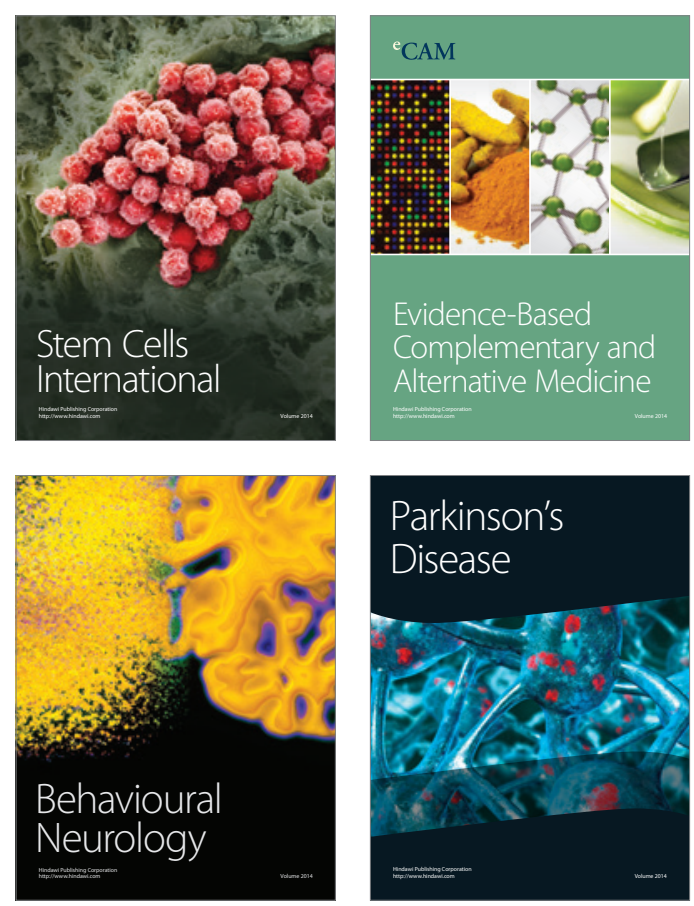

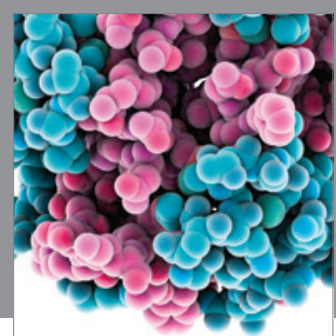

Journal of
Diabetes Research

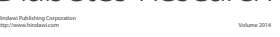

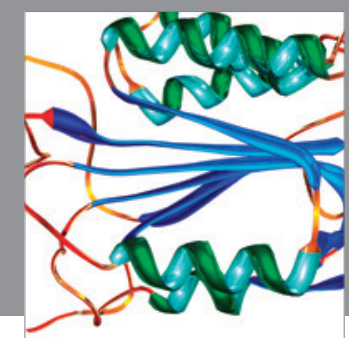

Disease Markers
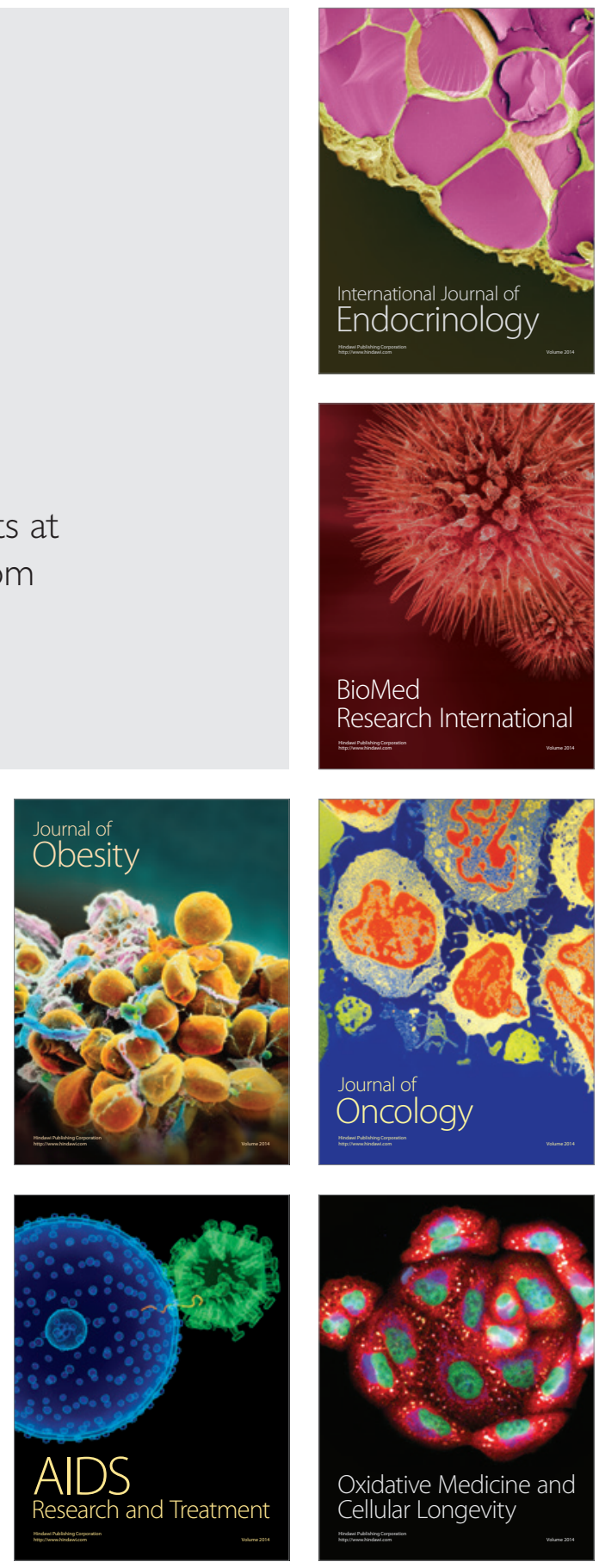